\title{
Experimental results to study superfluid correlations in a heated nucleus
}

\author{
Anatoly Sukhovoj ${ }^{1, a}$ and Liudmila Mitsyna ${ }^{1}$ \\ ${ }^{1}$ Frank Laboratory of Neutron Physics, Joint Institute for Nuclear Research, 141980 Dubna, Moscow region, Russia
}

\begin{abstract}
For 43 stable nuclei-targets in a wide interval of mass number $28 \leq A \leq 200$ the intensities $I_{\gamma \gamma}$ of the two-step cascades were measured. The experimental data are interpreted and approximated with a high accuracy within a simple practical model describing two-step $\gamma$ decay of neutron resonance.
\end{abstract}

\section{Introduction}

Up to now the main part of experimental data on the nuclear level density at the excitation energy lower than the nucleon binding energy $B_{n}$ are extracted from the experimental spectra of evaporated nucleons. The methods of data extraction are based on rather subjective ideas about partial emission widths of nuclear reaction products. As a result the values of level density extracted from evaporated nucleon's spectra are by 3-10 times higher than already measured level density at excitation energy $\sim 0.5 B_{n}$. Certainly, this is quite low accuracy.

To diminish essentially this fatal discrepancy is possible by using the experimental intensities of two-step $\gamma$ cascade for the simultaneous determination of both the level density and the partial widths.

The two nuclear properties, the level density and the partial widths of nuclear reaction emission products, have to be known in a very wide range of nuclear excitation energy if one likes to understand dynamics of superfluid correlations in excited nuclei Ref. [1]. In practice this demand can be fulfilled only by the measuring and analysis of the intensities of the two-step $\gamma$ cascades between a compound-state and some low-lying levels. Form of these data is determined by convolution of the energy dependence of level density $\rho\left(E_{e x}\right)$ and of the radiative widths $\Gamma\left(E_{\gamma}\right)\left(E_{e x}\right.$ is an excitation energy of a nucleus, $E_{\gamma}$ is a gamma-quantum energy). To determine $\rho$ and $\Gamma$ one is required to specify their functional forms with the minimum number of fitting parameters.

\section{The practical model of the process}

The model was developed and approved in FLNP JINR Refs. [2, 3]. The expression for the level density of fermion system $\rho_{l}$ is based on the model of n-quasiparticle

\footnotetext{
ae-mail: suchovoj@nf.jinr.ru
}

state density $\Omega_{n}[4]$

$$
\begin{gathered}
\rho_{l}=\frac{(2 J+1) \exp \left[-\frac{\left(J+\frac{1}{2}\right)^{2}}{2 \sigma^{2}}\right]}{2 \sqrt{2 \pi} \sigma^{3}} \Omega_{n}\left(E_{e x}\right) \\
\Omega_{n}\left(E_{e x}\right)=\frac{g_{n}\left(E_{e x}-U_{l}\right)^{n-1}}{((n / 2) !)^{2}(n-1) !}
\end{gathered}
$$

Here, $\sigma$ is a cut-off factor, $J$ is spin of a compound nucleus, $g$ is a density of single-particle states near the Fermi surface, $U_{l}$ is one of the required model parameters - a breaking energy of the $l$ th Cooper pair. Other model parameters $A_{l}, E_{\mu}, E_{v}$ and $\beta$ are included in the phenomenological coefficient $C_{\text {coll }}$ which describes the vibrational enhancement of level density at a given excitation energy $E_{e x}$.

$$
C_{\text {coll }}=A_{l} \exp \left(\sqrt{\frac{E_{e x}-U_{l}}{E_{v}}}-\frac{E_{e x}-U_{l}}{E_{\mu}}\right)+\beta .
$$

$A_{l}$ determines the absolute values of vibrational level density above the breaking point of each $l$ th Cooper pair; $E_{v}$ determines a rate of change of quasiparticle state energies; $E_{\mu}$ determines a rate of change of the nuclear entropy; the parameter $\beta \geq 1$.

To describe a smooth distribution of the $E 1$ and $M 1$ transition widths the model proposed by S.G. Kadmenskij, V.P. Markushev and W.I. Furman Ref. [5] was used with several (2-4) peaks of dipole strength functions. For each peak the most likely values of location, amplitude and slope of exponential decrease were found.

Thus, applying the fitting procedure with the method of maximum likelihood the following model parameters were determined:

1) the breaking thresholds $U_{l}$ up to $l=4$;

2) parameters $E_{\mu}$ and $E_{v}$ (the same for all Cooper pairs);

3 ) independent parameters $A_{l}$ for the density of vibrational levels above the breaking threshold $U_{l}$.

The process of decay of neutron resonance (or other compound state) $\lambda$ occurs by a two-step cascade through 
intermediate levels $i$ and some group of low-lying levels $f$. The extraction of parameters $\rho$ and $\Gamma$ from the intensities $I_{\gamma \gamma}\left(E_{1}\right)$ of two-step cascades for a given energy of the primary transition $E_{1}$ is complicated by need to solve the following system of nonlinear and strongly correlated equations:

$$
I_{\gamma \gamma}\left(E_{1}\right)=\sum_{\lambda, f} \sum_{i} \frac{\Gamma_{\lambda i}}{\Gamma_{\lambda}} \frac{\Gamma_{i f}}{\Gamma_{i}}=\sum_{\lambda, f} \frac{\Gamma_{\lambda i}}{\left\langle\Gamma_{\lambda i}\right\rangle m_{\lambda i}} n_{\lambda i} \frac{\Gamma_{i f}}{\left\langle\Gamma_{i f}\right\rangle m_{i f}},
$$

where $m_{\lambda i}$ is a number of initial primary transitions in energy intervals from the energy of primary level $\lambda$ to the intermediate level $i ; m_{i f}$ is a number of secondary $\gamma$ transitions in the energy intervals from the intermediate level $i$ to the lower level $f ; n_{\lambda i}$ is a number of intermediate levels involving in cascades in small energy intervals of initial transitions. To obtain the best values of model parameters it is necessary to determine a part of the primary transitions $I_{\gamma \gamma}\left(E_{1}\right)$ with a precision not worse than $10-20 \%$ in any energy interval.

\section{Examples of approximation}

The intensities $I_{\gamma \gamma}\left(E_{1}\right)$ of the two-step cascades were measured for 43 nuclei: even-odd nuclei ${ }^{71} \mathrm{Ge},{ }^{125} \mathrm{Te},{ }^{137} \mathrm{Ba}$, ${ }^{139} \mathrm{Ba},{ }^{163} \mathrm{Dy},{ }^{165} \mathrm{Dy},{ }^{181} \mathrm{Hf},{ }^{183} \mathrm{~W},{ }^{185} \mathrm{~W},{ }^{187} \mathrm{~W},{ }^{191} \mathrm{Os},{ }^{193} \mathrm{Os}$; even-even nuclei ${ }^{74} \mathrm{Ge},{ }^{114} \mathrm{Cd},{ }^{118} \mathrm{Sn},{ }^{124} \mathrm{Te},{ }^{138} \mathrm{Ba},{ }^{150} \mathrm{Sm}$, ${ }^{156} \mathrm{Gd},{ }^{158} \mathrm{Gd},{ }^{164} \mathrm{Dy},{ }^{168} \mathrm{Er},{ }^{174} \mathrm{Yb},{ }^{184} \mathrm{~W},{ }^{188} \mathrm{Os},{ }^{190} \mathrm{Os}$, ${ }^{196} \mathrm{Pt},{ }^{200} \mathrm{Hg}$; odd-odd nuclei ${ }^{28} \mathrm{Al},{ }^{40} \mathrm{~K},{ }^{52} \mathrm{~V},{ }^{60} \mathrm{Co},{ }^{64} \mathrm{Cu}$, ${ }^{128} \mathrm{I},{ }^{140} \mathrm{La},{ }^{160} \mathrm{~Tb},{ }^{166} \mathrm{Ho},{ }^{170} \mathrm{Tm},{ }^{176} \mathrm{Lu},{ }^{177} \mathrm{Lu},{ }^{182} \mathrm{Ta},{ }^{192} \mathrm{Ir}$, and ${ }^{198} \mathrm{Au}$. All these nuclei were investigated using our practical model. Variations of the initial fitting parameters and selection of approximations with minimal $\chi^{2}$ allow us to manage with 4 peaks of strength functions for nuclei ${ }^{28} \mathrm{Al},{ }^{64} \mathrm{Cu},{ }^{71} \mathrm{Ge},{ }^{177} \mathrm{Lu}$, and ${ }^{193} \mathrm{Os}$ and with 3 peaks for ${ }^{52} \mathrm{~V},{ }^{60} \mathrm{Co},{ }^{74} \mathrm{Ge},{ }^{156} \mathrm{Gd},{ }^{160} \mathrm{~Tb},{ }^{166} \mathrm{Ho},{ }^{176} \mathrm{Lu},{ }^{184} \mathrm{~W},{ }^{191} \mathrm{Os}$, and ${ }^{200} \mathrm{Hg}$. For the best fit of the data on remaining $28 \mathrm{nu}-$ clei one peak of the strength functions both of electric and of magnetic transitions was enough.

Fitting results with model calculations for 5 nuclei are presented in Figs. 1-5 as examples. These five Figures are organized as follows:

1. In the top panels, we display the following quantities as functions of the of primary transition energy $E_{1}$ : the sums of the experimental cascade intensities with their uncertainties as a histograms in $0.5 \mathrm{MeV}$ bins; the best fit for 6 different variants of approximation; the spectra calculated within nuclear statistical model with radiation strength function $k(M 1)=$ const.

2. In the middle panels, we show as functions of excitation energy $E_{e x}$ the most probable average density of intermediate levels of the two-step cascades in nuclei; level density calculated within the Fermi-gas model; partial level densities for the $l$ th Cooper pair at energy higher than the breaking threshold $U_{l}$.

3. In the bottom panel, we show, as functions of the of primary transition energy $E_{1}$, the strength functions of $E 1$ transitions and $M 1$-transitions; the sum of these strength

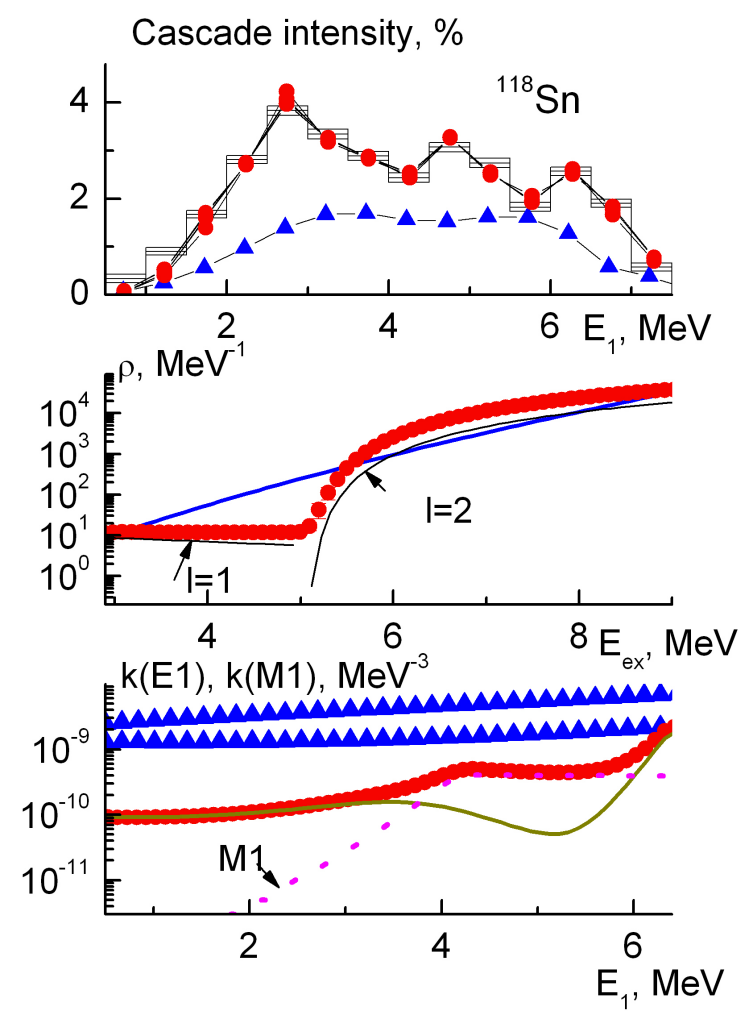

Figure 1. (Color online) Fitting and model calculations for ${ }^{118} \mathrm{Sn}$. Top panel: Histograms are the sums of the experimental cascade intensities; the best fit for 6 different variants of approximation - closed circles; spectra calculated within the nuclear statistical model with the radiative strength function $k(M 1)=$ const - triangles. Middle panel: the most probable average density of intermediate levels of the two-step cascades in nuclei - closed circles including errors; thin solid lines - partial level densities for the $l=1,2$.. Cooper pair at energy higher than the corresponding breaking threshold $U_{l}$; solid line - the Fermi-gas model calculation. Bottom panel: Strength functions $k(E 1)$ and $k(M 1)-$ solid line and dashed line, respectively; closed circles (include errors) - the sum of $E 1$ and $M 1$ strength functions; the upper band of triangles - the data of a giant $E 1$ resonance extrapolation; the lower band of triangles - calculations within the KMF model [5] with $k(M 1)=$ const.

functions; the data of the giant $E 1$ resonance extrapolations; results of calculations within the KMF model [5] with $k(M 1)=$ const

\section{Interpretation of the results}

Existence of the step-like structure in the nuclear level density demands in its turn the dependence of $\Gamma$ on the structure of initial and final nuclear levels of $\gamma$ transition. This is the only way to explain a smoothness of evaporated nucleon spectrum.

In Fig. 6, the best values of the parameters $E_{\mu}$ (the rate of change of the nuclear entropy) and $E_{v}$ (the rate of change of the quasiparticle energies) in all the nuclei are presented and compared with the mean pairing energy 


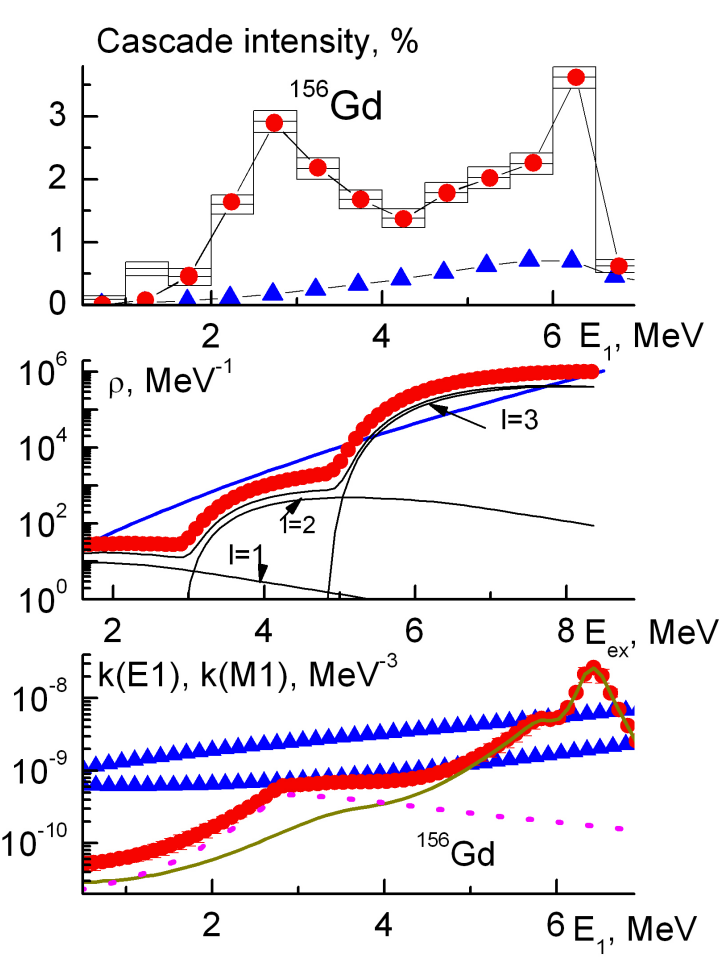

Figure 2. (Color online) Same as in Fig. 1 for ${ }^{156} \mathrm{Gd}$.

Cascade intensity, \%
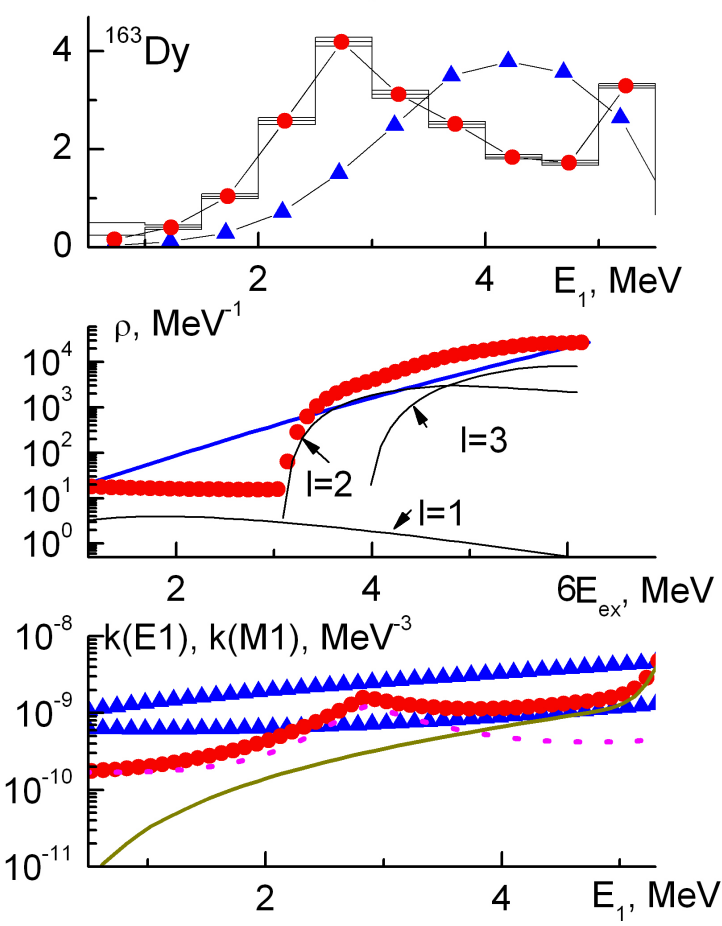

Figure 3. (Color online) Same as in Fig. 1 for ${ }^{163} \mathrm{Dy}$.

$\Delta_{0}=\frac{12.8}{\sqrt{A}} \mathrm{MeV}$ of the last nucleon in a nucleus with the mass number $A$.

In Fig. 7, the ratios $U_{l} / \Delta_{0}(l=2,3)$ and $B_{n} / \Delta_{0}$ are shown as functions of $A$. It is seen that in the mass region $150 \leq A \leq 190$, where most of the nuclei are deformed, the values $U_{l} / \Delta_{0}$ are noticeably lower than other. The parameter $U_{l}$ are determined with an accuracy allowing to notice the difference for nuclei with odd- and even numbers of neutrons (or protons). As evident from Fig. 7, for nuclei outside the aforementioned mass region the breaking threshold of the 3rd Cooper pair is about the neutron

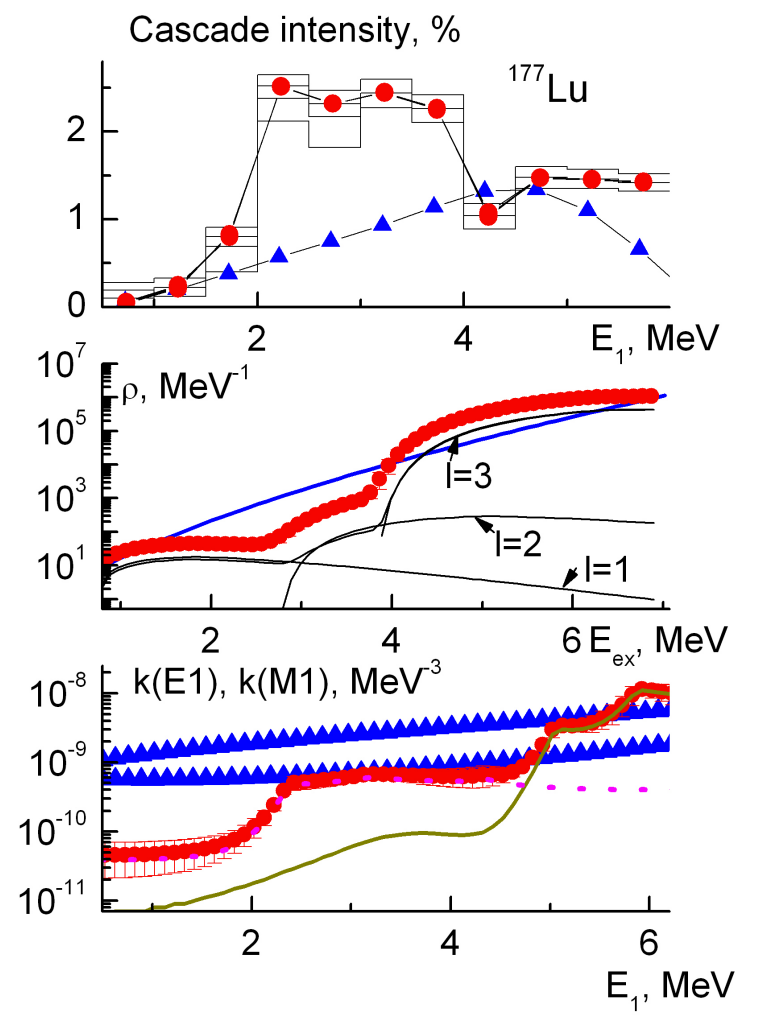

Figure 4. (Color online) Same as in Fig. 1 for ${ }^{177} \mathrm{Lu}$.

Cascade intensity, \%
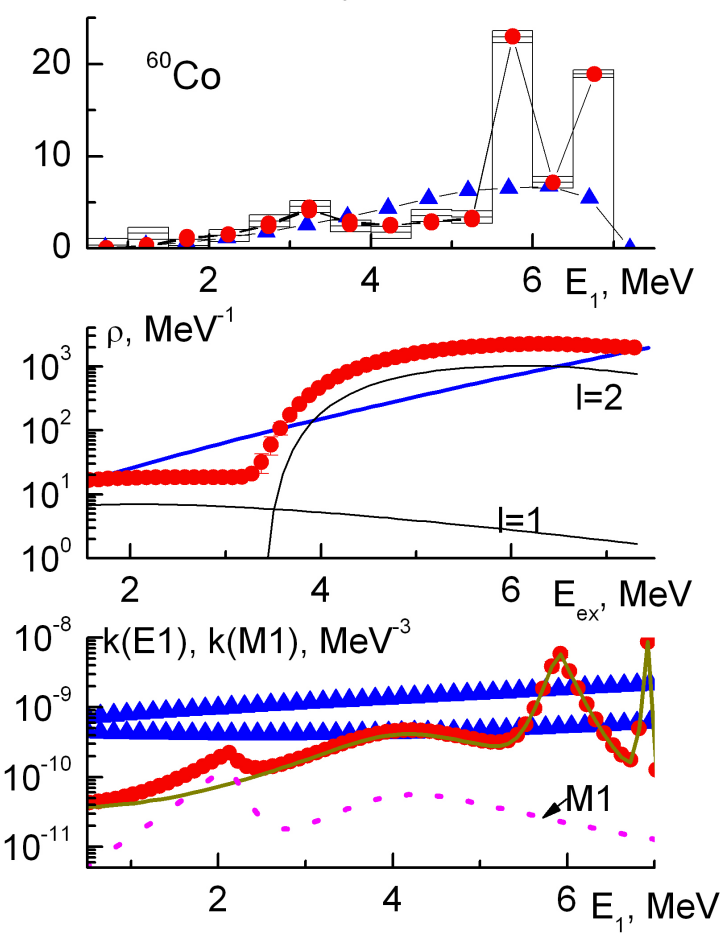

Figure 5. (Color online) Same in Fig. 1 for ${ }^{60} \mathrm{Co}$. 

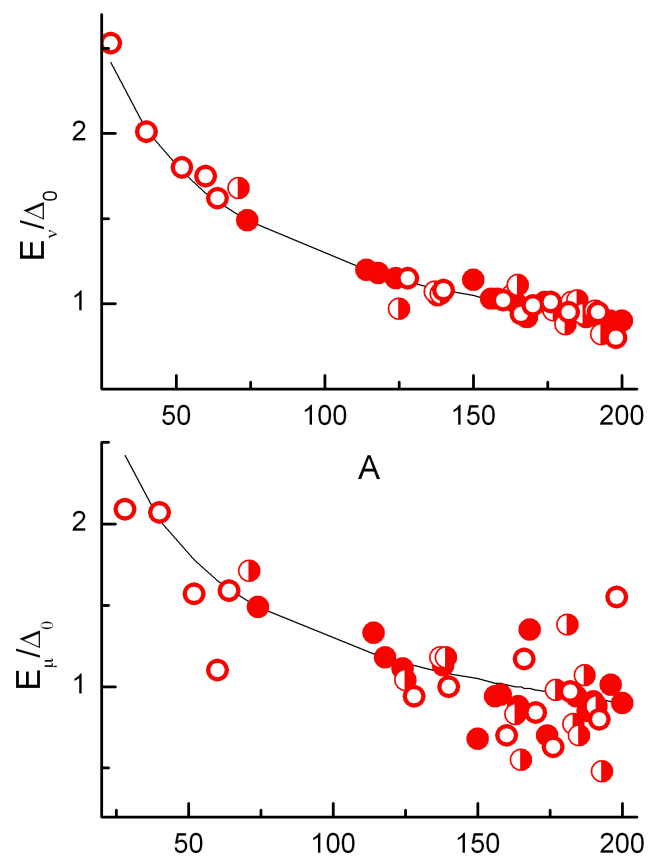

A

Figure 6. (Color online) Parameters $E_{\mu}$ and $E_{v}$ as functions of the mass number $A$. Closed circles - even-even nuclei, half-open circles - even-odd nuclei, and open circles - odd-odd nuclei. The line is the average value of the pairing energy of nucleon.

binding energy (in spherical nuclei this is a lower bound of the $U_{3}$ parameter). In deformed nuclei the $U_{3}$ value is twice as less (see squares in Fig. 7).

Thus, the main result of our analysis is a statement that the breaking thresholds of Cooper pairs are higher in spherical nuclei than in deformed ones.

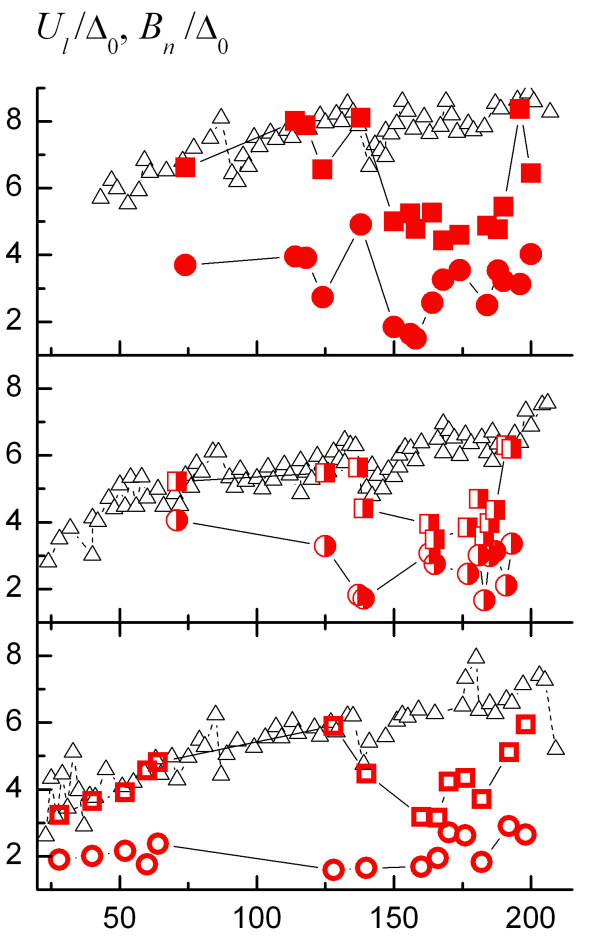

A

Figure 7. (Color online) Dependence of the breaking thresholds of the second (circles) and the third (squares) Cooper pairs on $A$. Closed symbols - even-even nuclei, half-open symbols - evenodd nuclei, and open circles - odd-odd compound nuclei. Open symbols - the mass dependence of the ratio $B_{n} / \Delta_{0}$.
To describe the measured parameters of the cascade gamma-decay of neutron resonances with the higher accuracy new theoretical models for $\rho$ and $\Gamma$ are needed. In these models, a dynamics of quasiparticles and phonon couplings starting from their minimal number should be taken into account. The parameters $U_{l}$ containing information about breaking thresholds of few Cooper pairs of nucleons (maybe including neutron-proton ones) can be used as a basis for a practical application of these models. The corresponding results will be of fundamental importance for understanding the nuclear superfluidity dynamics at excitation energies at least below the energy of giant dipole resonance.

Authors express their gratitude to the Organizing Committee of the NSRT15 Conference for the opportunity to present the results at the Conference.

\section{References}

[1] A.V. Ignatyuk, Report INDC-233(L). IAEA, Vienna, 1985

[2] A.M. Sukhovoj, Phys. At. Nucl. 78, 256 (2015)

[3] A.M. Sukhovoj, L.V. Mitsyna, Proceedings of the XXII Intern. Seminar on Interaction of Neutrons with Nuclei (Dubna, Russia, May 2014), Preprint JINR E3-2015-13, JINR, Dubna, 2015, p. 245.

[4] V.M. Strutinsky, Proceedings of the Intern. Congress on Nuclear Physics (Paris, France, 1958), Contributo Italiano Alla, Roma, 1959, p. 617; M. Herman and G. Reffo, Phys. Rev. C 36, 1546 (1987)

[5] S.G. Kadmenskij, V.P. Markushev, W.I. Furman, Sov. J. Nucl. Phys. 37, 165 (1983) 